\title{
Estimates of Weights in Gauss-Type Quadrature
}

\section{By E. L. Whitney}

1. Introduction. It may readily be verified that the angular distance $\Delta \theta=\theta_{i+1, n}-\theta_{i, n}$ between the zeros $\theta_{i, n}$ of the Legendre polynomial $P_{n}(\cos \theta)$ in $\cos \theta$ is roughly constant for large $n$. From the quadrature formula itself the weights may be estimated to a corresponding degree of accuracy. Direct asymptotic estimates of the weights corresponding to $\cos \theta=0$ in the $(2 n+1)$-point Gaussian quadrature are all available from Stirling's formula in the cases considered below. We here replace the $P_{n}$ by $C_{n}{ }^{\lambda}$, the Gegenbauer polynomials (effectively, tesseral harmonics or ultraspherical polynomials) of order $\lambda>0$, and the $H_{n}$ in the single limiting set of Hermite polynomials. Explicit formulas are derived: but the estimates for the general weights have a precision limited by the corresponding precision of the estimates of the zeros.

2. The Quadrature Formula. The Lagrange interpolation formula

$$
\begin{aligned}
f(x) & =\sum_{i} \frac{P(x) f\left(x_{i}\right)}{P^{\prime}\left(x_{i}\right)\left(x-x_{i}\right)}, \quad P\left(x_{i}\right)=0, \\
P^{\prime}\left(x_{i}\right) & \neq 0, \quad i=1,2, \cdots, n,
\end{aligned}
$$

algebraically valid for polynomials $f$ of degree $\nu<n$, the degree of $P$, has a rather limited direct use in polynomial approximation theory. Combined with various restrictions on $P$ to be in a basis of a set of polynomials with suitable properties, it becomes more useful.

Let $P^{*}(x)$ be of degree $n+1$, so that $P^{*}(x)=\operatorname{axP}(x)-b P(x)-c P_{*}(x)$ for constants $a, b$, and $c, P_{*}$ representing a polynomial of degree $\nu<n$. We set

$$
K(x, t)=K(t, x)=\frac{P^{*}(x) P(t)-P^{*}(t) P(x)}{x-t},
$$

a polynomial of degree $n$ in $x$ for each $t$, so that

$$
K(x, t)=a P(x) P(t)+c K_{*}(x, t),
$$

$K_{*}$ being defined in terms of $P$ and $P_{*}$ exactly as $K$ is determined by $P^{*}$ and $P$. In particular, $K(x, x)=P(x) P^{* \prime}(x)-P^{*}(x) P^{\prime}(x)$; and (1) is modified to become

$$
f(x)=\sum_{i} \frac{K\left(x, x_{i}\right)}{K\left(x_{i}, x_{i}\right)} f\left(x_{i}\right) .
$$

A suitable normalization with respect to a fixed integrable weight function $w$, essentially positive over the interval $I$ of integration, is

$$
\int_{I} K\left(x, x_{i}\right) w(x) d x=1
$$

so that (2) becomes

$$
\int_{I} f(x) w(x) d x=\sum_{i} f\left(x_{i}\right) W_{i},
$$

Received June 29, 1964. 
where

$$
W_{i}=\frac{1}{K\left(x_{i}, x_{i}\right)}
$$

is the formula for the weights.

From the above,

$$
K_{n}(x, t)=\sum_{i=0}^{n} a_{j} p_{j}(x) p_{j}(t)
$$

the indices $j$ indicating the degrees of the polynomials $\boldsymbol{p}_{j}$. Referring to (1), for example, we set

$$
p_{n}(t)=k_{n} t^{n}-\sum_{i=0}^{n-1} c_{j, n} p_{j}(t), \quad n=1,2, \dot{3}, \cdots,
$$

where

$$
\begin{gathered}
p_{0}(t)=k_{0}>0, \quad \int_{I} w(t) d t=\frac{1}{k_{0}^{2}} \\
\int_{I} p_{n}(t) p_{j}(t) w(t) d t=0, \quad 0 \leqq j<n,
\end{gathered}
$$

and

$$
\int_{I}\left\{p_{n}(t)\right\}^{2} w(t) d t=1
$$

The inductive definition is complete if we assume $k_{n}>0$. Indeed, for an arbitrary polynomial $P$,

$$
P(t)=\sum_{j=0}^{n} a_{j} k_{j} t^{j}=\sum_{j=0}^{n} a_{j, n} p_{j}(t),
$$

the $a_{j, n}$ being determined uniquely by the $a_{j}$ and $k_{j}$, where $\int_{I} k_{j} t^{j} p_{j}(t) w(t) d t=1$, so that

$$
x p_{n}(x)=\frac{k_{n}}{k_{n+1}} p_{n+1}(x)+b_{n} p_{n}(x)+\frac{k_{n-1}}{k_{n}} p_{n-1}(x)+\sum_{j=0}^{n-2} b_{j, n} p_{j}(x)
$$

in any case, with $b_{j, n}=0$ by (5). Then

$$
\begin{aligned}
K_{n}(x, t) & =\sum_{j=0}^{n} p_{j}(x) p_{j}(t) \\
& =\frac{k_{n}}{k_{n+1}} \frac{p_{n+1}(x) p_{n}(t)-p_{n}(x) p_{n+1}(t)}{x-t}, \quad \text { and } \\
K_{n}(x, x) & =\sum_{j=0}^{n}\left\{p_{j}(x)\right\}^{2} \\
& =\frac{k_{n}}{k_{n+1}}\left\{p_{n+1}^{\prime}(x) p_{n}(x)-p_{n}^{\prime}(x) p_{n+1}(x)\right\} \\
& =\int_{I}\left\{K_{n}(x, t)\right\}^{2} w(t) d t
\end{aligned}
$$

these being the standard Christoffel formulae (see [1]). 
If $f$ is of degree $2 n-1$ or less, the quotient $Q$ of $f$ by $p_{n}$ is uniquely determined, with remainder $p_{*}(t)=f(t)-Q(t) p_{n}(t)$ of degree $n-1$ or less. Then, if $p_{n}\left(x_{i}\right)=0$, $n$ being fixed,

$$
\begin{aligned}
\int_{I} p_{*}(t) w(t) d t & =\int_{I} f(t) w(t) d t, \quad \text { by }(5), \quad \text { and } \\
\int_{I} f(t) w(t) d t & =\sum_{i} W_{i} f\left(x_{i}\right),
\end{aligned}
$$

as before. (The formulae ( 7 ) guarantee the separation of $n$ distinct zeros in $I$.)

3. Sums of Squares. The Cesàro-one sums

$$
\sigma_{n}(x, t)=\frac{1}{n} \sum_{j=0}^{n-1} K_{j}(x, t)
$$

are expressed in the way suggested by Christoffel's method as follows:

$$
\begin{aligned}
n(x-t)^{2} \sigma_{n} & (x, t)=\sum_{j=0}^{n-1} \frac{k_{j}}{k_{j+1}}\left(b_{j}-b_{j+1}\right)\left\{p_{j+1}(x) p_{j}(t)+p_{j+1}(t) p_{j}(x)\right\} \\
& +\frac{k_{n-1}}{k_{n+1}}\left\{p_{n+1}(x) p_{n-1}(t)+p_{n-1}(x) p_{n+1}(t)\right\} \\
& -2\left(\begin{array}{c}
k_{n-1} \\
k_{n}
\end{array}\right)^{2} p_{n}(x) p_{n}(t)+2 \sum_{j=0}^{n-1} p_{j}(x) p_{j}(t)\left\{\left(\frac{k_{j}}{k_{j+1}}\right)^{2}-\left(\frac{k_{j-1}}{k_{j}}\right)^{2}\right\},
\end{aligned}
$$

where $b_{j}=\int_{I} t\left\{p_{j}(t)\right\}^{2} w(t) d t$ and $k_{-1}=0$.

Beginning with $k_{2}\left(b_{1}-b_{0}\right) / k_{1}=c_{1,2}$, we see that $b_{j}=b_{j+1}$ for all $j$ if and only if $w$ is symmetric over $I$. After a translation, we may assume in this case that the $p_{i}(t)$ are alternately even and odd polynomials. We assume that this condition holds in the sequel.

Let

$$
\Lambda_{j}(x)=\frac{k_{j-1}}{k_{j}} p_{j-1}(x)-\frac{k_{j}}{k_{j+1}} p_{j+1}(x),
$$

so that

$$
4 \frac{k_{j-1}}{k_{j+1}} p_{j+1}(x) p_{j-1}(x)=x^{2}\left\{p_{j}(x)\right\}^{2}-\left\{\Lambda_{j}(x)\right\}^{2} .
$$

Then, for suitable constants $c_{n}$, we set

$$
\begin{aligned}
L_{n}(x) & =\left(c_{n}{ }^{2}-x^{2}\right)\left\{p_{n}(x)\right\}^{2}+\left\{\Lambda_{n}(x)\right\}^{2} \\
& =4 \sum_{j=0}^{n-1}\left\{p_{j}(x)\right\}^{2}\left\{\left(\frac{k_{j}}{k_{j+1}}\right)^{2}-\left(\frac{k_{j-1}}{k_{j}}\right)^{2}\right\}+\left\{c_{n}{ }^{2}-4\left(\frac{k_{n-1}}{k_{n}}\right)^{2}\right\}\left\{p_{n}(x)\right\}^{2} .
\end{aligned}
$$

To make this formulation of sums of squares useful, the weight function $w$ is further restricted.

4. Gegenbauer Polynomials. See [1].

The expansion of $\rho^{-2 \lambda}=\left(1-2 r t+r^{2}\right)^{-\lambda}$ as a power series in $r$,

$$
(1-i z)^{-\lambda}(1-r \bar{z})^{-\lambda}=\sum_{j=0}^{\infty} C_{j}^{\lambda}(t) r^{j}
$$


subject to

$$
z+\bar{z}=2 t=2 \cos \theta, \quad z \bar{z}=1, \quad 0 \leqq r<1,
$$

determines the Gegenbauer polynomials $C_{n}{ }^{\lambda}$ of order $\lambda>0$. If $y$ is any successively differentiable function of $\rho$,

$$
r^{2} \frac{\partial^{2} y}{\partial r^{2}}+\frac{\partial^{2} y}{\partial t^{2}}=r^{2} \frac{d^{2} y}{d \rho^{2}}
$$

In the above case, $y=\rho^{-2 \lambda}$, so $d^{2} y / d \rho^{2}+((2 \lambda+1) / \rho)(d y / d \rho)=0$, and so

$$
r^{2} \frac{\partial^{2} y}{\partial r^{2}}+(2 \lambda+1) r \frac{\partial y}{\partial r}+\left(1-t^{2}\right) \frac{\partial^{2} y}{\partial t^{2}}=(2 \lambda+1) t \frac{\partial y}{\partial t} .
$$

Comparing coefficients in the power series, we have

$$
\frac{d}{d t}\left\{\left(1-t^{2}\right)^{\lambda+1 / 2} \frac{d C_{n}^{\lambda}(t)}{d t}\right\}=-n(n+2 \lambda)\left(1-t^{2}\right)^{\lambda-1 / 2} C_{n}^{\lambda}(t) .
$$

Multiplying by $C_{j}^{\lambda}(t)$, alternating the indices $n$ and $j$, and subtracting, then integrating from $t=-1$ to $t=1$, we have

$$
C_{j}^{\lambda}(t)=\sqrt{ } h_{j} p_{j}(t),
$$

the $\left\{p_{j}\right\}$ being orthogonal (with property (5)) with respect to $w$,

$$
w(t)=\left(1-t^{2}\right)^{\lambda-1 / 2} \text {. }
$$

Here,

$$
\int_{-1}^{+1}\left\{C_{j}^{\lambda}(t)\right\}^{2} w(t) d t=h_{j},
$$

easily calculated explicitly. From the definition above, using the series and the binomial theorem,

$$
C_{n}^{\lambda}(\cos \theta)=\sum_{j=0}^{n}\left(\begin{array}{c}
\lambda+j-1 \\
j
\end{array}\right)\left(\begin{array}{c}
\lambda+n-j-1 \\
n-j
\end{array}\right) \cos (\overline{n-2 j \theta}),
$$

so

$$
\left|C_{n}{ }^{\lambda}(t)\right| \leqq C_{n}{ }^{\lambda}(1)=\left(\begin{array}{c}
2 \lambda+n-1 \\
n
\end{array}\right), \quad-1 \leqq t \leqq 1,
$$

if $\lambda>0$.

We may make direct use of the Christoffel formulae (7), comparison of terms in a linear expansion, and induction, to obtain

$$
\begin{aligned}
2 h_{n} k_{0}{ }^{2}(n+\lambda) & =\lambda\left(\begin{array}{c}
n+2 \lambda-1 \\
n
\end{array}\right), \\
4\left(\frac{k_{n-1}}{k_{n}}\right)^{2} & =\frac{n(n+2 \lambda-1)}{(n+\lambda)(n-1+\lambda)},
\end{aligned}
$$


and

$$
\lambda !^{2} 2^{2 \lambda}(n+\lambda)\left\{{C_{n}}^{\lambda}(t)\right\}^{2}=\pi\left(\begin{array}{c}
2 \lambda+n-1 \\
n
\end{array}\right) \lambda(2 \lambda) !\left\{p_{n}(t)\right\}^{2}
$$

Also,

$$
\frac{k_{n-1}}{k_{n}} p_{n-1}(0)=-\frac{k_{n}}{k_{n+1}} p_{n+1}(0)
$$

so that

$$
\lim _{n \rightarrow \infty}\left\{p_{2 n}(0)\right\}^{2}=\frac{2}{\pi}
$$

and

$$
\lim _{n \rightarrow \infty} p_{n}(1)(n+\lambda)^{-2 \lambda}=\sqrt{\frac{2}{\pi}} \frac{2^{\lambda} 2 !}{(2 \lambda) !},
$$

the relative errors in the corresponding approximations being of (order) $O\left(1 /(n+\lambda)^{2}\right)$ uniformly in $n$ for fixed $\lambda$ by Stirling's formula.

We set $z=\left(1-t^{2}\right)^{\lambda / 2} p_{n}(t)$, and find

$$
\frac{d z}{d t}=(n+\lambda)\left(1-t^{2}\right)^{\lambda / 2-1} \Lambda_{n}(t)
$$

using (6) and (11). If

$$
L_{n}(t)=\left\{p_{n}(t)\right\}^{2}\left(1-t^{2}\right)+\left\{\Lambda_{n}(t)\right\}^{2},
$$

(11) becomes

$$
\frac{d}{d t}\left\{L_{n}(t)\left(1-t^{2}\right)^{\lambda-1}\right\}=-\frac{2 \lambda(1-\lambda)}{n+\lambda}\left(1-t^{2}\right)^{\lambda-2} p_{n}(t) \Lambda_{n}(t) .
$$

From the above quadratic relation, and (6),

$$
2 \sqrt{ }\left(1-t^{2}\right)\left|p_{n}(t) \Lambda_{n}(t)\right| \leqq L_{n}(t) .
$$

Differentiating the logarithm of $L_{n}$, and integrating, we have

$$
\log \left\{\frac{L_{n}(t)}{L_{n}(0)}\left(1-t^{2}\right)^{\lambda-1}\right\}<\frac{|\lambda(1-\lambda)|}{n+\lambda} \frac{|t|}{\sqrt{ }\left(1-t^{2}\right)}, \quad 0<|t|<1 .
$$

In particular, $\lim _{n \rightarrow \infty} L_{n}(t)\left(1-t^{2}\right)^{\lambda-1}=2 / \pi,-1<t<1$.

However, relation (10) now reads as follows:

$$
L_{n}(t)=-2 \sum_{j=0}^{n-1} \frac{\lambda(1-\lambda)\left\{p_{j}(t)\right\}^{2}}{(j-1+\lambda)(j+\lambda)(j+1+\lambda)}-\frac{\lambda(1-\lambda)\left\{p_{n}(t)\right\}^{2}}{(n+\lambda-1)(n+\lambda)},
$$

whence

$$
\begin{aligned}
L_{n}(t)\left(1-t^{2}\right)^{\lambda-1}= & \left\{p_{n}(t)\right\}^{2}\left(1-t^{2}\right)^{\lambda}+\left\{\Lambda_{n}(t)\right\}^{2}\left(1-t^{2}\right)^{\lambda-1} \\
= & \frac{2}{\pi}-\frac{\lambda(1-\lambda)\left\{p_{n}(t)\right\}^{2}\left(1-t^{2}\right)^{\lambda-1}}{(n+\lambda)(n+\lambda+1)} \\
& +2 \sum_{j=n+1}^{\infty} \frac{\lambda(1-\lambda)\left\{p_{j}(t)\right\}^{2}\left(1-t^{2}\right)^{\lambda-1}}{(j-1+\lambda)(j+\lambda)(j+1+\lambda)}
\end{aligned}
$$


The maximum of $z^{2}=\left\{p_{n}(t)\right\}^{2}\left(1-t^{2}\right)^{\lambda}$ in any subinterval of $I$ with endpoints $t=x_{i}$ or $t= \pm 1$, corresponds only to $\Lambda_{n}(t)=0$, so that if

$$
\begin{aligned}
(n+\lambda)(n+\lambda+1)\left(1-t^{2}\right) & \geqq \frac{|\lambda(1-\lambda)|}{\epsilon}, \\
p_{n}(t)\left(1-t^{2}\right)^{\lambda}(1 \pm \epsilon) & <\frac{2}{\pi},
\end{aligned}
$$

and, otherwise,

$$
p_{n}(1)\left(1-t^{2}\right)^{\lambda}
$$

is uniformly bounded, by (12) and (13).

On the other hand, if $p_{n}\left(x_{i}\right)=0$,

$$
\left\{\Lambda\left(x_{i}\right)\right\}^{2}\left(1-x_{i}{ }^{2}\right)^{\lambda-1}=\frac{2}{\pi}+\frac{2 \lambda(1-\lambda)}{1-x_{i}{ }^{2}} \sum_{j=n+1}^{\infty} \frac{\left\{p_{j}\left(x_{i}\right)\right\}^{2}\left(1-x_{i}{ }^{2}\right)^{\lambda}}{(j-1+\lambda)(j+\lambda)(j+1+\lambda)},
$$

where

$$
\sum_{j=n+1}^{\infty} \frac{\left\{p_{j}(x)\right\}^{2}\left(1-x^{2}\right)^{\lambda}}{(j-1+\lambda)(j+\lambda)(j+1+\lambda)}<\frac{1+\epsilon_{n}{ }^{\prime}}{\pi(n+\lambda)^{2}}
$$

and $\lim _{n \rightarrow \infty} \epsilon_{n}{ }^{\prime}=0$, if $| \pm 1+x|>\delta$, any fixed positive number. That is, if $\left| \pm 1+x_{i}\right|>\delta$

$$
\frac{1}{W_{i}}=K_{n}\left(x_{i}, x_{i}\right)=\frac{\Lambda_{n}\left(x_{i}\right) p_{n}^{\prime}\left(x_{i}\right)}{2}=\frac{n+\lambda}{2} \frac{\left\{\Lambda_{n}\left(x_{i}\right)\right\}^{2}}{1-x_{i}{ }^{2}},
$$

and for such zeros $x=x_{i}$,

$$
W_{i} \cong \frac{\pi}{n+\lambda}\left(1-x_{i}{ }^{2}\right)^{\lambda},
$$

with a relative-error estimate

$$
\frac{|\lambda(1-\lambda)|}{(n+\lambda)^{2}\left(1-x_{i}^{2}\right)}
$$

for both upper and lower bounds.

If $n$ is an odd number, and $x_{i}=0$, we easily compute

$$
\frac{1}{W_{i}}=\frac{n+\lambda}{\pi}\left\{1+\frac{\lambda(1-\lambda)}{2 n^{2}}+\frac{\lambda(1-\lambda)^{2}}{n^{3}}+\cdots\right\},
$$

using Stirling's formula, for the corresponding median weight $W_{i}$. The precision of the estimate here is easily controlled; but in the general case the sums of squares seem difficult to handle with precision.

5. Spacing of Zeros. Let $v=p_{n}{ }^{\prime}(t) / p_{n}(t)$. Using (11), we find

$$
\left(1-t^{2}\right) \frac{d v}{d t}=(2 \lambda+1) t v-n(n+2 \lambda)-\left(1-t^{2}\right) v^{2} .
$$

Combining this with the Christoffel formulae, using induction and the result $\left|p_{n}(t)\right| \leqq p_{n}(1)$, we have

$$
v \leqq \frac{p_{n}^{\prime}(1)}{p_{n}(1)}=\frac{n(n+2 \lambda)}{2 \lambda+1} \quad \text { if } \quad x_{n}<t \leqq 1,
$$


$x=x_{n}$ being the zero of $p_{n}(t)$ nearest $t=1$. Since $\left(p_{n}\left(x_{n}\right)-p_{n}(1)\right) /\left(x_{n}-1\right)<$ $p_{n}{ }^{\prime}(1)$, we have $x_{n}<1-(2 \lambda+1) /(n(n+2 \lambda))$.

In general, if we set $t=\sin \phi$, the equivalent differential relation

$$
\begin{aligned}
&-\frac{d}{d \phi}\left\{\arctan \left[\frac{\Lambda_{n}(t)}{p_{n}(t) \sqrt{ }\left(1-t^{2}\right)}\right]\right\} \\
&=n+\lambda+\frac{\lambda(1-\lambda)}{n+\lambda} \frac{\left\{p_{n}(t)\right\}^{2}}{L_{n}(t)}, \quad x_{i}<t<x_{i+1},
\end{aligned}
$$

gives us the necessary information concerning the spacing of the zeros. We have

$$
\pi=\Delta \arctan \left[\frac{\Delta_{n}(t)}{p_{n}(t) \sqrt{ }\left(1-t^{2}\right)}\right]=(n+\lambda) \Delta \phi_{i}+\frac{\lambda(1-\lambda)}{n+\lambda} \int_{\phi_{i}}^{\phi_{i+1}} \frac{\left\{p_{n}(t)\right\}^{2}}{L_{n}(t)} d \phi,
$$

where $x_{i}=\sin \phi_{i}$ and $\Delta \phi_{i}=\phi_{i+1}-\phi_{i}$.

6. Hermite Polynomials. From the defining formulas, we easily obtain

$$
\left(\frac{d}{d t}\right)^{m}\left\{C_{n}^{\lambda}(t)\right\}=2^{n}\left(\begin{array}{c}
\lambda+m-1 \\
m
\end{array}\right) C_{n-m}^{\lambda+m}(t)
$$

by induction on $m$. Among other results, relations between the tesseral harmonics of Legendre,

$$
\begin{gathered}
P_{n}^{(m)}(t)=\left(1-t^{2}\right)^{m / 2}\left(\frac{d}{d t}\right)^{m}\left\{P_{n}(t)\right\}, \\
P_{n}(t)=C_{n}^{\lambda}(t) \text { for } \lambda=\frac{1}{2},
\end{gathered}
$$

and the Gegenbauer polynomials follow. Formally, the trigonometric basis is given by $\lambda=0$ and $\lambda=1$.

If $t^{2}=s^{2} / 2 \lambda, s$ being fixed, and $\lambda \rightarrow \infty$, we have

$$
w(t) \rightarrow e^{-s^{2} / 2} \text {. }
$$

For the bounded $n$ and $s$,

$$
C_{n}^{\lambda}(t) \vec{n} H_{n}(s), \quad \text { if } \quad \lambda \rightarrow \infty,
$$

the corresponding Hermite polynomial.

Let

$$
\frac{d}{d t}\left\{H_{n}(t) e^{-t^{2} / 2}\right\}=-H_{n+1}(t) e^{-t^{2} / 2}, \quad H_{0}(t)=1,
$$

for $n=0,1,2, \cdots$. Then

$$
H_{n}{ }^{\prime}(t)=n H_{n-1}(t)
$$

by Leibnitz' rule for successive differentiation. It follows immediately that

$$
H_{n}(x)=\sum_{j<(n+1) / 2}\left(\begin{array}{l}
n \\
2 j
\end{array}\right)(-1)^{j} C_{j} x^{n-2,}
$$


for a single set of coefficients $\left\{C_{j}\right\}$. Since

$$
t H_{n}(t)=n H_{n-1}(t)+H_{n+1}(t)
$$

from the pair of relations given above, we have the Christoffel formulae

$$
H_{n}(x, t)=\sum_{j=0}^{n} \frac{H_{j}(x) H_{j}(t)}{j !}=\frac{H_{n+1}(x) H_{n}(t)-H_{n}(x) H_{n+1}(t)}{n !(x-t)}
$$

and

$$
\begin{aligned}
H_{n}(x, x) & =\sum_{j=0}^{n} \frac{H_{j}{ }^{2}(x)}{j !}=\frac{(n+1) H_{n}^{2}(x)-n H_{n+1}(x)}{n !} . \\
& =\frac{1}{\sqrt{ }(2 \pi)} \int_{-\infty}^{\infty} H_{n}^{2}(x, t) e^{-t^{2} / 2} d t .
\end{aligned}
$$

To arrive at the last result, we make use of

$$
\int_{-\infty}^{\infty} \int_{-\infty}^{\infty} e^{-\left(x^{2}+t^{2}\right) / 2} d x d t=2 \pi
$$

or the limits given above. Since

$$
\sqrt{ }(2 \pi) e^{-x^{2} / 2}=\int_{-\infty}^{\infty} e^{-t^{2} / 2+i x t} t^{n} d t
$$

we have

$$
\sqrt{ }(2 \pi) H_{n}(x) e^{-x^{2} / 2}=(-i)^{n} \int_{-\infty}^{\infty} e^{-t^{2} / 2+i x t} t^{n} d t .
$$

Let $z=e^{-t^{2} / 4} H_{n}(t)$, so that

$$
\frac{d z}{d t}=e^{-t^{2} / 4}\left\{n H_{n-1}(t)-\frac{t}{2} H_{n}(t)\right\}
$$

and

$$
\frac{d^{2} z}{d t^{2}}=-z\left(n+\frac{1}{2}-\frac{t^{2}}{4}\right)
$$

Then

$$
(t z)^{2}-4\left(\frac{d z}{d t}\right)^{2}=4 n e^{-t^{2} / 2} H_{n-1}(t) H_{n+1}(t),
$$

so that

$$
e^{-x^{2} / 2}\left\{\sum_{j=0}^{n-1} \frac{H_{j}{ }^{2}(x)}{j !}+\frac{1}{2} \frac{H_{n}{ }^{2}(x)}{n !}\right\}=\sum_{j=0}^{n-1} \frac{H_{j}{ }^{2}(0)}{j !}+\frac{1}{2} \frac{H_{n}{ }^{2}(0)}{n !}-\frac{1}{2} \int_{0}^{x} t e^{-t^{2} / 2} \frac{H_{n}{ }^{2}(t)}{n !} d t
$$

from the Christoffel formula. We do not obtain different results from the formulation of the Cesàro-one sums, in this case. We define

$$
L_{n}(x)=\frac{1}{\sqrt{ } n}\left\{\sum_{j=0}^{n-1} \frac{H_{j}^{2}(x)}{j !}+\frac{1}{2} \frac{H_{n}^{2}(x)}{n !}\right\},
$$


so that

$$
\lim _{n \rightarrow \infty} L_{n}(0)=\sqrt{ } \frac{2}{\pi}
$$

Then, also,

$$
L_{n}(x) e^{-x^{2} / 2}=L_{n}(0)-\frac{1}{2 \sqrt{ } n} \int_{0}^{x} t \frac{H_{n}^{2}(t)}{n !} e^{-t^{2} / 2} d t
$$

so here

$$
\sqrt{ } n L_{n}(t) e^{-t^{2} / 2}=\frac{1}{n !}\left\{\left(\frac{d z}{d t}\right)^{2}+\left(n+\frac{1}{2}-\frac{t^{2}}{4}\right) z^{2}\right\}
$$

and

$$
\lim _{n} L_{n}(x) e^{-x^{2} / 2}=\sqrt{ } \frac{2}{\pi} .
$$

The formula for the weights $W_{i}$ corresponding to $H_{n}\left(x_{i}\right)=0$ becomes

$$
\frac{1}{W_{i}}=H_{n}\left(x_{i}, x_{i}\right)=\sqrt{ } n L_{n}\left(x_{i}\right),
$$

so

$$
W_{i} \cong \sqrt{\frac{\pi}{2 n}} e^{-x_{i}^{2} / 2}
$$

with a relative error estimate

$$
\frac{x_{i}^{2}}{2 n-\delta} \text { if } x_{i}{ }^{2}<2(1+\delta) .
$$

If we consider the Fourier sine expansion over the interval $(a, a+\pi / k)$ between zeros $x=a, x=b=a+\pi / k$, of $H_{n}(x) e^{-x^{2} / 4}$, we have

$$
\int_{a}^{b}\left\{\left(\frac{d z}{d t}\right)^{2}-k^{2} z^{2}\right\} d t>0
$$

Now

$$
\int_{a}^{b}\left\{\left(\frac{d z}{d t}\right)^{2}-\left(n+\frac{1}{2}-\frac{t^{2}}{4}\right) z^{2}\right\} d t=0
$$

so that

$$
b-a>\frac{2 \pi}{\sqrt{ }\left(4 n+2-a^{2}\right)} .
$$

Otherwise, $d z / d t<0$ if $t^{2} \geqq 4 n+2$. We cannot have $z=0$ there, since $z>0$ if $t \rightarrow \infty$ for fixed $n$. Then

$$
b^{2}<4 n+2
$$


We may point out that the estimates, for Cesàro-one and related sums, remain useful in establishing convergence properties of the expansions of functions (e.g., of bounded variation) as series of orthogonal polynomials.

University of Alberta

Edmonton, Alberta

Canada,

1. A. Erdély et Al., Higher Transcendental Functions, Vol. II, McGraw-Hill, New York, 1953, Chapter 10, p. 174. MR 15, 419. 